\title{
1 The Development of Crystal Growth Technology
}

\author{
HANS J. SCHEEL \\ SCHEEL CONSULTING, CH-8808 Pfaeffikon SZ, Switzerland
}

\begin{abstract}
The industrial production of crystals started with A. Verneuil with his flamefusion growth method 1902. He can be regarded as the father of crystal growth technology as his principles of nucleation control and crystal-diameter control are adapted in most later growth methods from the melt, like Tammann, Stöber, Bridgman, Czochralski, Kyropoulos, Stockbarger, etc. The important crystal pulling from melts named after Czochralski was effectively developed by Teal, Little and Dash.

The multi-disciplinary nature of technology of crystal and epilayer fabrication, the complex multi-parameter processes - where ten or more growth parameters have to be compromised and optimized, and also the scaling problem have impeded the scientific development of this important area. Only recently has the numerical simulation of Czochralski melts started to become useful for growth technologists, the deep understanding of the striation problem allowed the experimental conditions to grow striation-free crystals to be established, and the control of epitaxial growth modes permitted the preparation of atomically flat surfaces and interfaces of importance for the performance of opto-electronic and superconducting devices.

Despite the scale of the multi-billion dollar crystal and epilayer fabrication and crystal-machining industry and the annual need worldwide of at least 400 engineers, there is so far no formation of specialists for crystal production, epitaxy technology, crystal machining and surface preparation. A special curriculum is required due to the multi-disciplinary character of crystal-growth technology (CGT) which does not fit into a single classical university discipline like chemical, mechanical, materials, or electrical engineering, or crystallography, thermodynamics, solid-state physics, and surface physics. The education scheme for CGT has to include all these disciplines and basic sciences to such an extent that the finished engineers and scientists are capable of interacting and collaborating with specialists from the various disciplines. It is up to the interested industries to request CGT engineers from the technical universities and engineering schools.
\end{abstract}


Crystal-growth technology and epitaxy technology had developed along with the technological development in the 20th century. On the other hand, the rapid advances in microelectronics, in communication technologies, in medical instrumentation, in energy and space technology were only possible after the remarkable progress in fabrication of large, rather perfect crystals and of large-diameter epitaxial layers (epilayers). Further progress in CGT and education of CGT engineers is required for significant contributions to the energy crisis. High-efficiency white light-emitting diodes for energy-saving illumination and photovoltaic/thermo-photovoltaic devices for transforming solar and other radiation energy into electric power with high yield depend on significant advances in crystal growth and epitaxy technology. Also, the dream of laserfusion energy and other novel technologies can only be realized after appropriate progress in the technology of crystal and epilayer fabrication.

\subsection{HISTORICAL INTRODUCTION}

Fundamental aspects of crystal growth had been derived from early crystallization experiments in the 18th and the 19th century (Elwell and Scheel 1975, Scheel 1993). Theoretical understanding started with the development of thermodynamics in the late 19th century (Gibbs, Arrhenius, Van't Hoff) and with the development of nucleation and crystal growth theories and the increasing understanding of the role of transport phenomena in the 20th century. The phenomena of undercooling and supersaturation and the heat of crystallization were already recognized in the 18th century by Fahrenheit and by Lowitz. The corresponding metastable region, the existence range of undercooled melts and solutions, was measured and defined in 1893/1897 by Ostwald and in 1906 by Miers, whereas the effect of friction on the width of this Ostwald-Miers region was described in 1911 and 1913 by Young. Although the impact of stirring on this metastable region is important in mass crystallization of salt, sugar and many chemicals, it is not yet theoretically understood.

The rates of nucleation and crystallization in glasses were the foundation to nucleation theories. The crystal surface with steps and kinks of Kossel in 1927 allowed Stranski and Kaishew in 1934 to define the work of separation of crystal units as repeatable steps as the basis of the first crystal-growth theories. With the understanding of facet formation as a function of the entropy of fusion in 1958 by Jackson, and depending on the density of bonds in the crystal structure 1955 by Hartman and Perdok, the role of screw dislocations as continuous step sources in the formation of growth hillocks (Frank 1949), and with the generalized crystal growth theory of Burton, Cabrera and Frank 1951, many growth phenomena could be explained.

In the growth of crystals from a fluid medium (melt, solution, gas phase) the heat and mass transport phenomena also play a significant role, as was observed early by Rouelle 1745 and Frankenheim 1835. The diffusion boundary layer 
defined by Noyes and Whitney 1897 was used in the growth-rate equation of Nernst 1904 and confirmed by interferometric measurements of concentration profiles around growing crystals by Berg 1938 and by others. Forced convection was recognized to be beneficial for diffusion-limited growth by Wulff 1886, Krüger and Finke 1910, and Johnsen 1915 for open systems with stirrers, whereas smooth stirring in sealed containers can be achieved with the accelerated crucible rotation technique ACRT of Scheel 1971/1972. The growth of inclusion-free crystals from the melt can be accomplished by observing the principles of "diffusional undercooling" of Ivantsov 1951 and "constitutional supercooling" of Tiller et al. 1953. Formation of inclusions, i.e. growth instability, can be prevented in growth from solutions by sufficient flow against or along the crystal facets: Carlson 1958 developed an empirical theory which was utilized by Scheel and Elwell 1972 to derive the maximum stable growth rate and optimized programming of supersaturation for obtaining large inclusion-free crystals.

Microscopic and macroscopic inhomogeneities in doped crystals and in solid solutions are caused by segregation phenomena, which are related to mass and heat transfer. Based on the derivation of effective distribution coefficients for melt growth by Burton et al. (1953) and by van Erk (1982) for growth from solutions, the theoretical and experimental conditions for growth of striation-free crystals could be established (Rytz and Scheel 1982, Scheel and Sommerauer 1983, Scheel and Swendsen 2001).

There have been remarkable developments with respect to size and perfection of crystals, with silicon, sapphire, alkali and earth alkali halides reaching diameters up to $0.5 \mathrm{~m}$ and weights of nearly $500 \mathrm{~kg}$. These advances in Czochralski, Kyropoulos, heat-exchanger method, and Bridgman-Stockbarger growth were accompanied by numerical simulations which have become increasingly powerful to predict the optimized conditions. However, further advances in computer modelling and in the reliability of the used physico-chemical data are required in order to increase the efficiency and precision of computer simulations and to allow the prediction of the best crystal-growth technology including growth parameters for the growth of new large and relatively perfect crystals.

\subsection{THE DEVELOPMENT OF CRYSTAL-GROWTH METHODS}

Industrial crystal production started with Verneuil 1902 who with the flame-fusion growth process named after him achieved for the first time control of nucleation and thus single crystals of ruby and sapphire with melting points above $2000{ }^{\circ} \mathrm{C}$. By formation of the neck and the following enlargement of the crystal diameter, not only was the seed selection achieved in a crucible-free process, but also the structural perfection of the growing crystal could be controlled to some extent.

Figure 1.1 shows the stages of Verneuil growth of high-melting oxides, and Figure 1.2 the relatively simple apparatus where powder is fed into the hydrogen-oxygen flame and enters the molten cap of first the cone and neck, and 


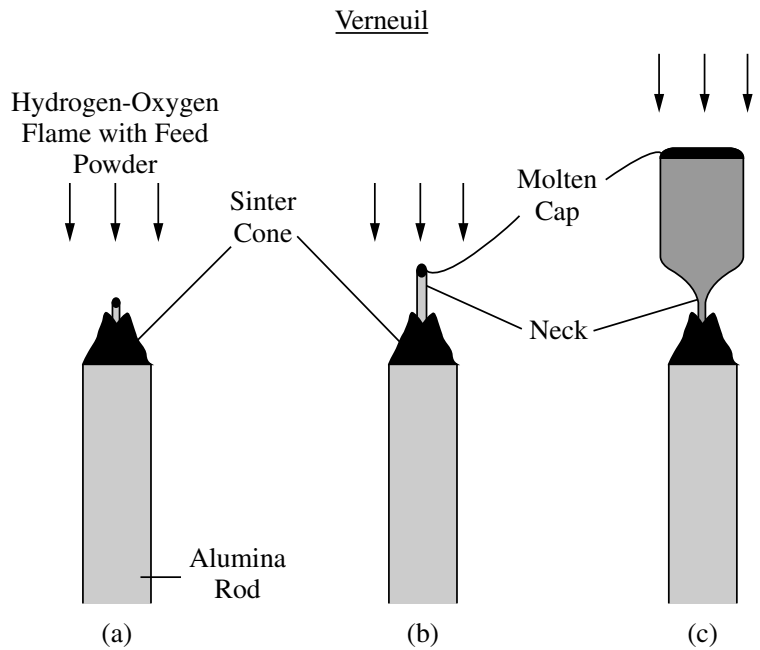

Figure 1.1 Stages of flame-fusion (Verneuil) growth of ruby, schematic: (a) formation of sinter cone and central melt droplet onto alumina rod, (b) growth of the neck by adjustment of powder supply and the hydrogen-oxygen flame, (c) Increase of the diameter without overflow of the molten cap for the growth of the single-crystal boule. (Reprinted from H. J. Scheel, J. Cryst. Growth 211(2000) 1-12, copyright (2000) with permission from Elsevier Science.)

then the molten surface layer of the growing crystal. The latter is slowly moved downwards so that the surface-melting conditions remain within the observation window. Historical details of Verneuil's development were given by Nassau 1969 and Nassau and Nassau 1971. The industrialization for production of ruby watchstones and synthetic gemstones started with Verneuil's laboratory in Paris in 1910 with 30 furnaces and with the Djevahirdjian factory at Monthey VS in Switzerland where the CIBA chemical company could provide hydrogen. The annual ruby production reached 5 million carats in 1907, and 10 million carats ruby and 6 million carats sapphire in 1913. Nowadays, the production capacity of sapphire for watch windows and other applications is 250 tons $\left(1.25 \times 10^{9}\right.$ carats $)$, the largest fraction from the Djevahirdjian factory with 2200 Verneuil furnaces, and this with the practically unchanged process.

The principles of the Verneuil method with nucleation, growth rate and diameter control have been applied in most of the growth processes described in the following years: Tammann 1914, Stöber 1925, Bridgman 1923, Stockbarger 1936, vertical-gradient-freeze growth of Ramsberger and Malvin 1927, and in the Czochralski process 1918/1950, see Figure 1.3 and Wilke-Bohm 1988. Thus, Verneuil can really be regarded as the founder of crystal-growth technology.

However, the most important method of fabrication of semiconductor and oxide crystals consists of pulling crystals from melts contained in crucibles, a method named after Czochralski. The origin of this started with the topical area 


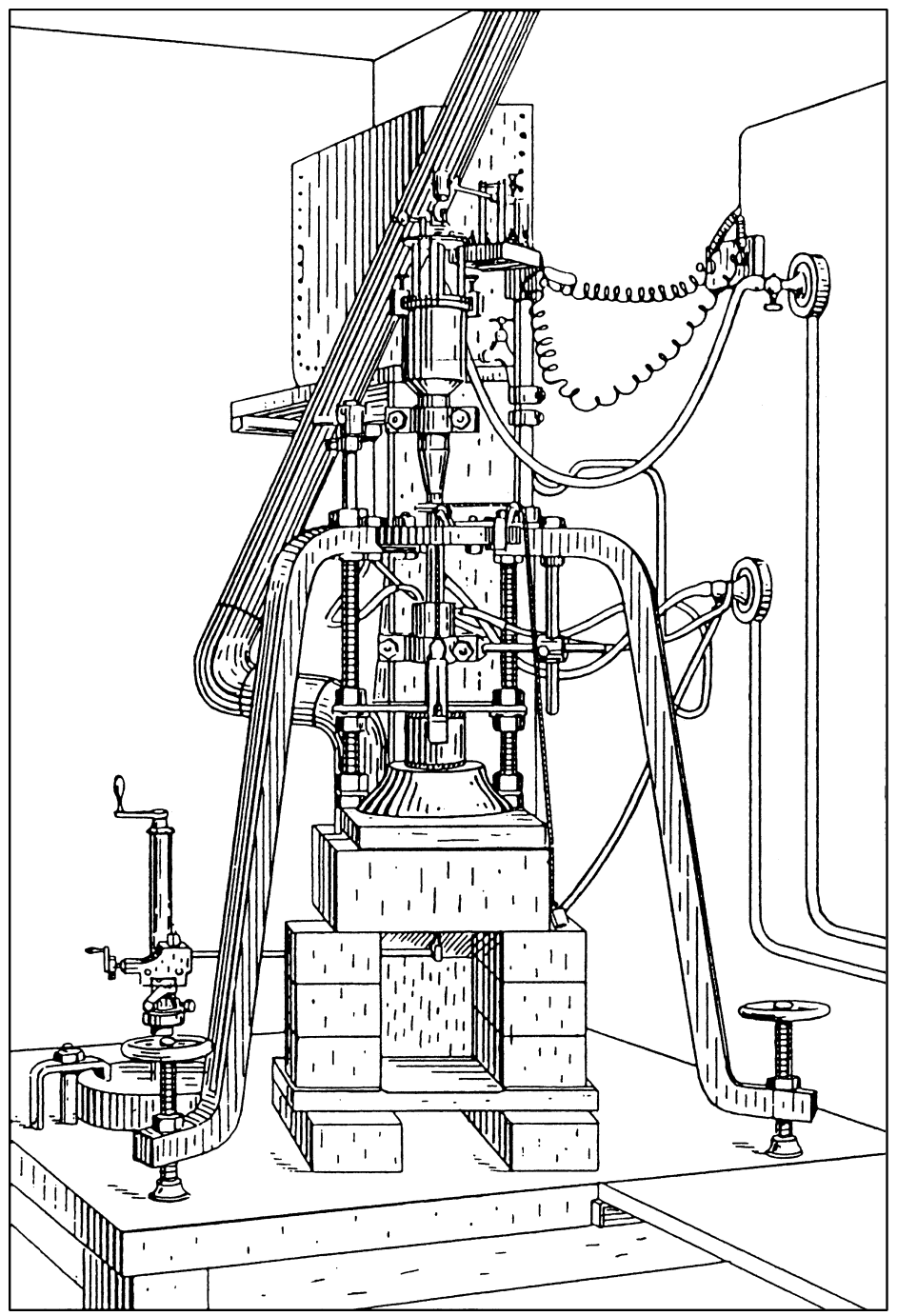

Figure 1.2 The relatively simple apparatus of Verneuil for flame-fusion growth. (Reprinted from D. T. J. Hurle (ed.) Handbook of Crystal Growth 1, Fundamentals, Part A, Chapter 1, 1993, copyright (1993) with permission from Elsevier Science.)

of physical chemistry for 50 years, the measurement or crystallization velocities, which was initiated by Gernez 1882, who had found a high crystallization rate of molten phosphorus. After Tammann's studies of glass crystallization, Czochralski presented in 1918 "a new method for measurement of the crystallization velocity of metals" where he pulled fibers of low-melting metals from melts. Soon after this, von Gomperz used floating dies to control the fiber diameter 

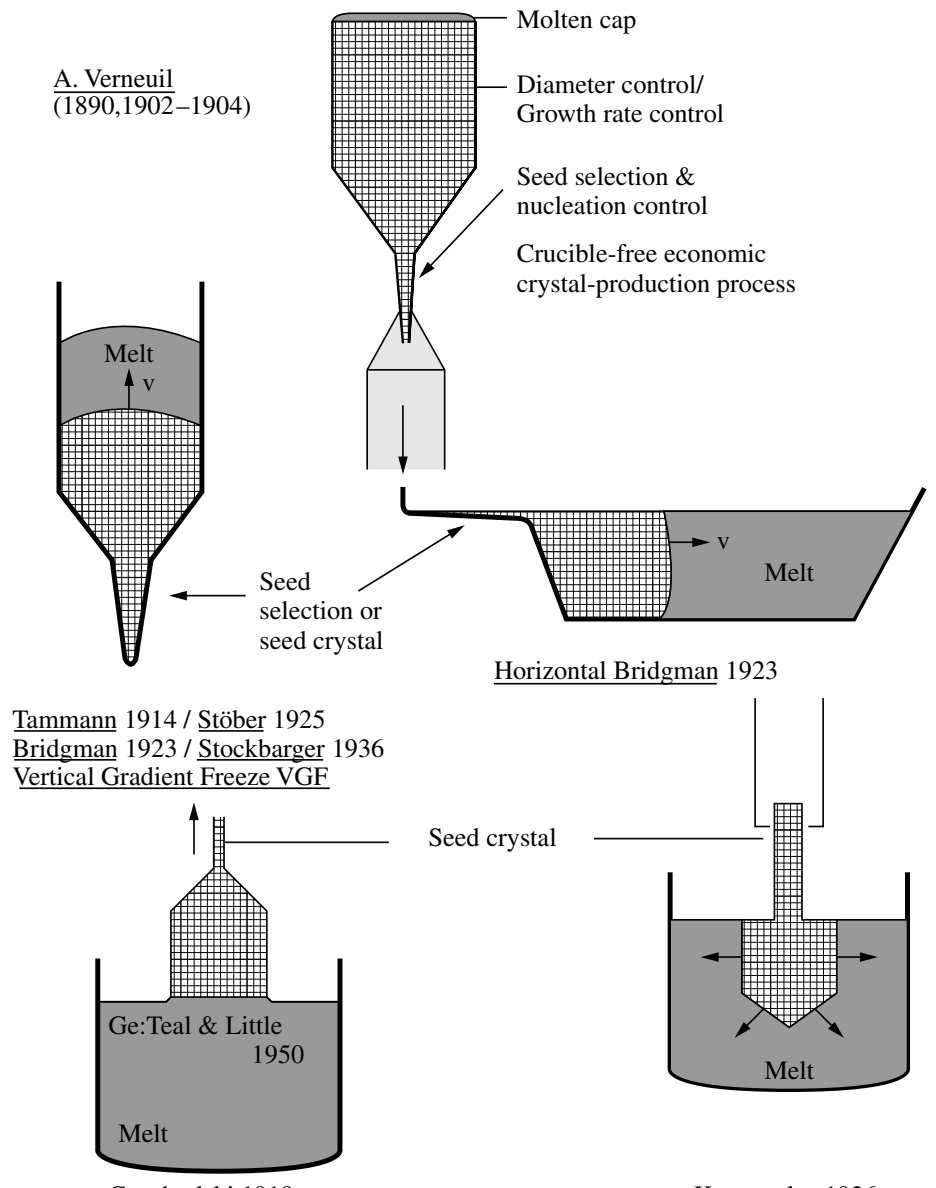

Czochralski 1918

Kyropoulos 1926

Figure 1.3 Modification of Verneuil's principles of nucleation control and increasing crystal diameters in other crystal-growth techniques. (Reprinted from H. J. Scheel, J. Cryst. Growth 211(2000) 1-12, copyright (2000) with permission from Elsevier Science.)

(an early Stepanov or EFG process) 1921, and in 1928 Kapitza measured crystallization rates and prepared single-crystalline bismuth in a vertical glass tube (early zone melting).

Czochralski never considered pulling a crystal for research, although he could have adapted the inverse Verneuil principle, which was well known at that time. The metal crystals for research in Czochralski's laboratory were prepared by the Bridgman method. Therefore, it is no wonder that Zerfoss, Johnson and Egli in their review on growth methods at the International Crystal Growth Meeting in Bristol UK 1949 mentioned Verneuil, Bridgman-Stockbarger, Stöber and Kyropoulos techniques, but not Czochralski. 
With the discovery of the transistor at Bell Laboratories there arose a need for single crystals of semiconductors, first of germanium. Teal and Little invented and developed crystal pulling of relatively large Ge crystals in 1950. Then it was the book of Buckley in 1951, who erroneously named pulling of large crystals after Czochralski, and this name became widespread with the fast-growing importance of crystal pulling. Possibly it is too late to change the name to Teal-Little-Dash, the latter due to his contribution to dislocation-free crystals by optimized seed and neck formation. The development of Czochralski and liquid-encapsulated Czochralski growth was discussed by Hurle 1987.

The majority of crystals is produced by pulling from the melt. Crystal dimensions have reached $10 \mathrm{~cm}$ for $\mathrm{InP}, \mathrm{LiTaO}_{3}$, sapphire and other oxide crystals, $15 \mathrm{~cm}$ for $\mathrm{LiNbO}_{3}$ and $\mathrm{GaAs}$, and 30 to $40 \mathrm{~cm}$ for silicon and for halide scintillator crystals with melt dimensions up to $1 \mathrm{~m}$. Numerical modelling has assisted these developments, for instance for the optimization of the crystal and crucible rotation rates and of the temperature distribution and the design of heaters, afterheaters and heat shields. However, for the axial segregation and the striation problems the final general solution has not yet been established. The following approaches to reduce convection have been partially introduced in crystal production: double crucible for GaAs and stoichiometric $\mathrm{LiNbO}_{3}$, magnetic fields in various configurations for semiconductors, and shallow melts or small melt volumes with continuous feeding for silicon and for halide scintillator crystals: these approaches are discussed in this volume. An alternative approach to solve, or at least minimize, the axial segregation and the striation problem is the co-rotating ring Czochralski (CRCZ) method which induces a hydrodynamic double-crucible effect: by optimized rotation rate of crystal and co-rotating ring (inserted into the melt) and counter-rotation rate of the crucible, a nearly convection-free melt fraction is achieved below the growing crystal, whereas the larger fraction of the melt is homogenized by combined forced and natural convection (Scheel 1995).

Only a few Czochralski-grown elements like silicon, germanium, and copper are produced dislocation-free by application of necking as developed by Dash in 1959 . Most crystals have dislocations of typically $10^{3}$ to $10^{6} \mathrm{~cm}^{-2}$ due to the generally large temperature gradients at the growing interface and due to the postgrowth defect agglomeration behind the growth front (Völkl and Müller 1989). Attempts to grow with low-temperature gradients at the Institute of Inorganic Chemistry in Novosibirsk or to grow the crystal into the melt by the Kyropoulos method (SOI-method in St. Petersburg) have yielded low dislocation crystals of, for example, sapphire up to $40 \mathrm{~cm}$ diameter, but have not yet been developed for large-scale production. However, alternative technologies like Bridgman and vertical gradient freeze (VGF) have gained importance for production of semiconductor crystals of improved structural perfection like GaAs, InP, and CdTe and its solid solutions. In vertical Bridgman and in VGF growth the forced convection by the accelerated-crucible rotation technique (ACRT) of Scheel 1972 has increased homogeneity, structural perfection, and stable growth rates in the cases of halides (Horowitz et al. 1983) and doped CdTe (Capper 1994, 2000). 
In silicon, the octahedral void defects (see Falster and Itsumi in this volume) and their distribution as well as oxygen and dopant homogeneity require further understanding (see Abe, Falster) and process optimization, otherwise an increasing fraction of silicon wafers have to be provided with epitaxial layers in order to achieve the homogeneity of surface properties required for highly integrated microelectronic circuit structures.

Examples of other remarkable developments in the growth of bulk crystals are briefly given: Hydrothermal growth of large high-quality low-dislocationdensity quartz by VNIISIMS/Russia and Sawyer/USA, hydrothermal growth of large $\mathrm{ZnO}$ and emerald crystals, high-temperature solution growth of perovskites and garnets by top-seeded solution growth TSSG by Linz et al. and by the accelerated crucible rotation technique (ACRT) by Scheel and by Tolksdorf, TSSG growth of KTP and CLBO (the latter by Sasaki and Mori), aqueoussolution growth of KDP (Sasaki, Zaitseva), Bridgman-Stockbarger growth of alkali halides and of $\mathrm{CaF}_{2}$, float-zone growth of $\mathrm{TiO}_{2}$ rutile by Shindo et al., skull-melting growth of zirconia by Wenckus and Osiko, and Czochralski growth of numerous oxide compounds mainly in US, French, Japanese and recently Korean and Taiwan companies.

\subsection{CRYSTAL-GROWTH TECHNOLOGY NOW}

The world crystal production is estimated at more than 20000 tons per year, of which the largest fraction of about $60 \%$ are semiconductors silicon, GaAs, InP, $\mathrm{GaP}, \mathrm{CdTe}$ and its alloys. As can be seen in Figure 1.4, optical crystals, scintillator crystals, and acousto-optic crystals have about equal shares of $10 \%$, whereas laser and nonlinear-optic crystals and crystals for jewelry and watch industry have shares of a few $\%$ only. This scale of crystal production and the fact that most
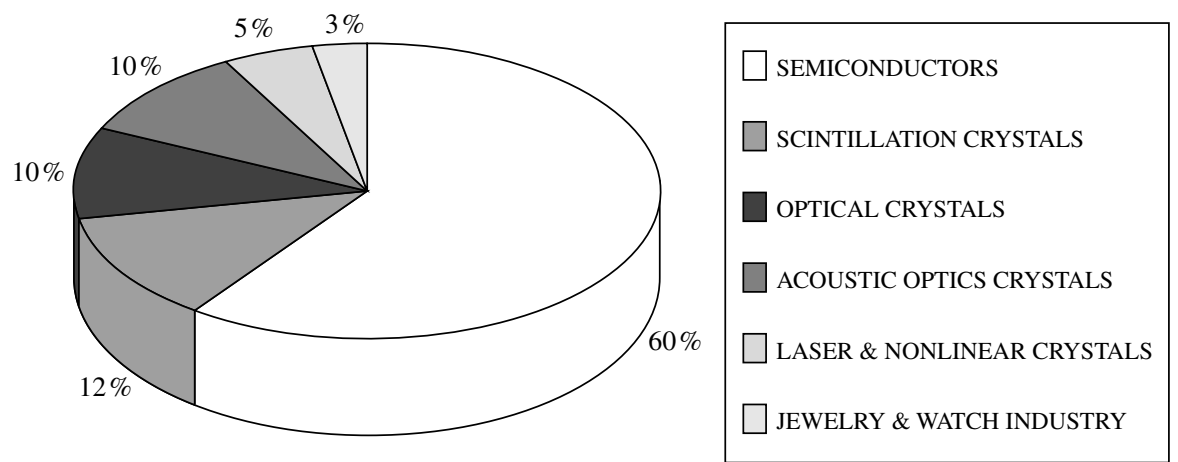

Figure 1.4 Estimated shares of world crystal production in 1999. (Reprinted from H. J. Scheel, J. Cryst. Growth 211(2000) 1-12, copyright (2000) with permission from Elsevier Science.) 
crystals are produced in factories specialized in silicon or $\mathrm{GaAs}$ or $\mathrm{LiNbO}_{3}$, etc. has caused an increasing degree of specialization. Furthermore, crystal growth has split into five major areas so that cross-fertilization and communication are more and more reduced:

1. Fundamental theoretical and experimental crystal growth studies.

2. Laboratory crystal growth for preparing research samples.

3. Industrial fabrication of single crystals and their machining and characterization.

4. Fabrication of metallic/dendritic crystals (e.g. turbine blades).

5. Mass crystallization (salt, sugar, chemicals).

Epitaxy has similarly separated from bulk crystal growth and has split into:

1. Fundamental/theoretical and experimental epitaxy studies and surface phenomena.

2. Epitaxy growth methods (VPE, MOCVD/MOVPE, MBE, ALE, LPE).

3. Materials classes (GaAs, InP, GaN, II-VI compounds, high- $T_{\mathrm{c}}$ superconductors, low-dimensional/quantum-well/quantum-dot structures, etc.).

As a consequence of these separations and splits, numerous specialized conferences, workshops and schools are established and regularly organized, new specialized journals appear, and the common language gets more and more lost, even to the extent that formerly well-defined terms are misused.

The situation is critical in research and development involving crystal growth and epitaxy. Due to a worldwide lack of education in these areas, frequently growth methods are employed for problems for which they are not well-suited or even not suited at all. It is postulated (Scheel 2002) that for a specific crystal of defined size and perfection there is only

\section{one optimum and economic growth technology}

considering thermodynamics, growth kinetics, and economic factors. This aspect is schematically shown in Figure 1.5, where the crystal performance, the crystal size, the efficiency of the growth method, and the price of crystal are shown. The shape factor may play a role when, for instance, special shapes are needed in the application, for example square, rectangular or oval watch faces of sapphire. The concept of one or maximum two optimum technologies should be considered for industrial crystal fabrication, whereas in laboratories the existing equipment or experience may determine a nonoptimum growth method. This postulate holds obviously also for epitaxial methods: for example, high- $T_{\mathrm{c}}$ compounds have been prepared by $>1000$ groups (after the discovery of high-temperature superconductivity, HTSC) by vapour-phase epitaxy, which cannot yield the atomically flat surfaces required for reliable HTSC tunnel-device technology. Instead only liquid 


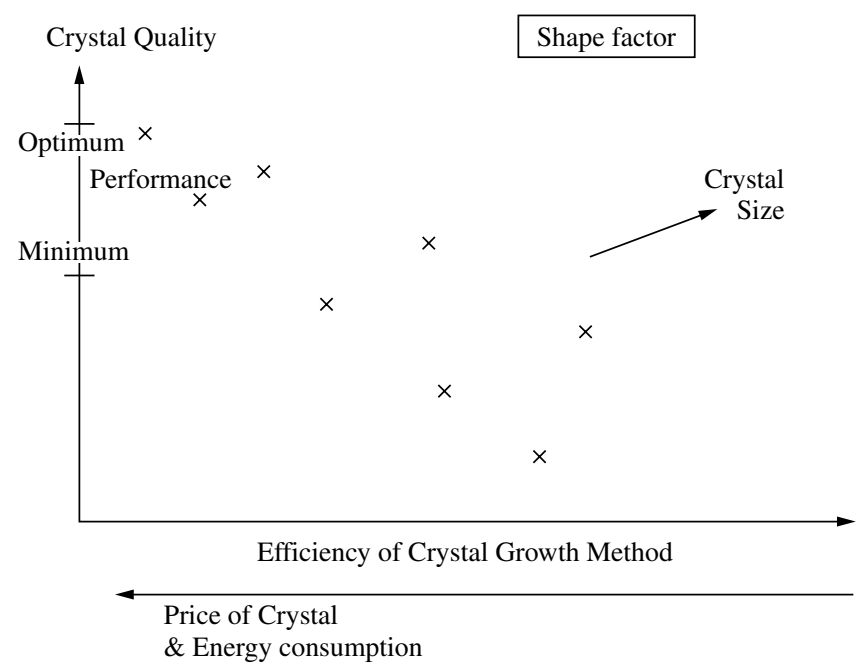

Figure 1.5 Schematic diagram of the efficiencies of various crystal-growth methods and the achieved crystal sizes and crystal quality. Note that there is normally only one optimum economic technology for the required crystal performance and size.

phase epitaxy near thermodynamic equilibrium can give atomically flat surfaces, as discussed elsewhere in this volume. Here, it could be added that the lack of recognition of the materials problems and specifically of the crystal growth and epitaxy problems have caused tremendous economic (and intellectual) losses not only in industries but also in research. As a recent prominent example, HTSC as a whole could not be properly developed, neither its physical understanding nor its many predicted applications because the chemical and structural complexity of the not very stable HTSC compounds were neglected. Most physical HTSC measurements were nonreproducible because the used crystals and layers were not sufficiently characterized for those structural and chemical defects that have an impact on these measurements or applications.

We realize that 'education' is needed for the important field of crystal-growth technology in order to achieve efficient developments in novel high technologies, in energy saving, in solar energy generation, in future laser-fusion energy and with a better approach in HTSC: in all these areas where crystal-growth technology is the progress-determining factor (Scheel 2002). However, in view of the multidisciplinary nature of crystal growth technology it will be not easy to initiate a curriculum in a classical university with the rather strict separation of disciplines in faculties or departments. In addition, the specialized education in CGT can start only after the multidisciplinary basic education and requires additional courses of 1 to 2 years. Furthermore, there are no teachers for this field as most crystal growers are specialized and experts in specific materials or specific methods only. In addition education in crystal growth technology and 
Table 1.1 Complexity of crystal growth technology CGT

a. Multi-Disciplinary

- Chemistry (all fields) including Chemical Engineering

- Materials Science \& Engineering

- Mechanical \& Electrical Engineering (especially hydrodynamics, machine design, process control)

- Theoretical Physics (especially thermodynamics, non-equilibrium thermodynamics, statistical mechanics)

- Applied Crystallography and Crystal Chemistry

- Solid-State Physics

b. Complexity

- Phase Transformation from Fluid (melt, solution, vapour) to Crystal

- Scaling Problem: Control of surface on $\mathrm{nm}$ scale in growth system of $\sim \mathrm{m}$ size, hampers numerical simulation

- Complex Structure \& Phenomena in Melts and Solutions

- Multi-Parameter Processes: Optimize and compromise 10 parameters

There is only one Optimum and Economic Growth Technology for a Specific Size and Performance of Crystal or Crystalline Layer!

epitaxy technology should be complemented by practical work and by periods in industries: another obstacle where, however, interested industries will collaborate. This practical work is needed in order to understand and appreciate the complexity (see Table 1.1) of crystal-growth processes in view of the involved phase transformation, of the many parameters that have to be compromised and optimized, and in view of the scaling problem, which hampers realistic numerical simulation.

\subsection{CONCLUSION}

Crystal-growth technology (CGT) including epilayer fabrication technology is of greatest importance for energy saving, for renewable energy, and for novel high technologies. Therefore the education of CGT engineers and CGT scientists is needed as well as significant support and publicity. Excellent students with intuition and motivation can have a great impact in crystal-growth technology and thus contribute to the energy problem and to high-technology developments of mankind.

\section{REFERENCES}

Buckley H. E. (1951), Crystal Growth, Wiley, New York.

Burton J. A., Prim R. C. and Slichter W. P. (1953), J. Chem. Phys. 21, 1987.

Capper P. (1994), Prog. Crystal Growth Charact. 28, 1. 
Capper P. (2000), at second International School on Crystal Growth Technology ISCGT-2 Mount Zao, Japan August 24-29, 2000 (book in preparation).

Elwell D. and Scheel H. J. (1975), Crystal Growth from High-Temperature Solutions, Academic Press, London- New York, (reprint foreseen 2003).

Horowitz A., Gazit D., Makovsky J. and Ben-Dor L. (1983), J. Cryst. Growth 61, 323 and Horowitz A., Goldstein M. and Horowitz Y., J. Cryst. Growth 61, 317.

Hurle D. T. J. (1987), J. Cryst. Growth 85, 1.

Ivantsov G. P. (1951, 1952), Dokl. Akad. Nauk SSSR 81, 179; 83, 573.

Nassau K. (1969), J. Cryst. Growth 5, 338.

Nassau K. and Nassau J. (1971), Lapidary J. 24, 1284, 1442, 1524.

Rytz D. and Scheel H. J. (1982), J. Cryst. Growth 59, 468.

Scheel H. J. (1972), J. Cryst. Growth 13/14, 560.

Scheel H. J. (1993/1994), Historical Introduction, Chapter 1 in Handbook of Crystal Growth, editor D. T. J. Hurle, Vol. 1 Elsevier, Amsterdam.

Scheel H. J. (1995), US Patent No. 5471943.

Scheel H. J. (2002), Plenary lecture at Second Asian Conference on Crystal Growth and Crystal Technology CGCT-2 August 28-31, 2002 in Seoul, Korea, (proceedings in preparation).

Scheel H. J. and Elwell D. (1972), J. Cryst. Growth 12, 153.

Scheel H. J. and Sommerauer J. (1983), J. Cryst. Growth 62, 291.

Scheel H. J. and Swendsen R. H. (2001), J. Cryst. Growth 233, 609.

Tiller W. A., Jackson K. A., Rutter J. W. and Chalmers B. (1953), Acta Met. 1, 428.

van Erk W. (1982), J. Cryst. Growth 57, 71.

Völkl J. and Müller G. (1989), J. Cryst. Growth 97, 136.

Wilke K.-Th. and Bohm J. (1988) Kristallzüchtung, VEB Deutscher Verlag der Wissenschaften, Berlin. 